\title{
GABAergic Presubicular Projections to the Medial Entorhinal Cortex of the Rat
}

\author{
Theo van Haeften, Floris G. Wouterlood, Barbara Jorritsma-Byham, and Menno P. Witter \\ Graduate School for Neurosciences Amsterdam, Research Institute Neurosciences Vrije Universiteit, Department of \\ Anatomy and Embryology, Amsterdam, The Netherlands
}

\begin{abstract}
We characterized presubicular neurons giving rise to bilateral projections to the medial entorhinal cortex (MEA) of the rat. Retrograde labeling of presubiculo-entorhinal projections with horseradish peroxidase and subsequent GABA immunocytochemistry revealed that $20-30 \%$ of the ipsilaterally projecting neurons are GABAergic. No GABAergic projections to the contralateral MEA were observed. GABAergic projection neurons were observed only in the dorsal part of the presubiculum, which, when taking into account the topography of presubicular projections to MEA, indicates that only the dorsal part of MEA receives GABAergic input. The GABAergic projection neurons constitute $\sim 30-40 \%$ of all GABAergic neurons present in the superficial layers of the dorsal presubiculum. Using doublelabel fluorescent retrograde tracing, we found that the ipsilateral and contralateral presubiculo-entorhinal projections originate from different populations of neurons. Anterograde labeling of presubiculo-entorhinal projections and electron mi-
\end{abstract}

croscopical analysis of labeled terminals substantiated the presence of a restricted GABAergic presubiculo-entorhinal projection. A small fraction of afferents to only ipsilateral dorsal MEA formed symmetrical synapses with dendritic shafts. No symmetrical synapses on spines were noted. Most afferents to the dorsal part of ipsilateral MEA, as well as all afferents to the remaining ipsilateral and contralateral MEA, formed asymmetrical synapses with both spines and dendritic shafts in an almost equal ratio. Thus, we conclude that the majority of the presubiculo-entorhinal projections exert an excitatory effect on both principal neurons and interneurons. The projections from the dorsal part of the presubiculum comprise a small inhibitory component that originates from GABAergic neurons and targets entorhinal interneurons.

Key words: presubiculum; GABA; projection neurons; retrograde tracing; double-fluorescence tracing; electron microscopy; feedforward disinhibition
The presubiculum is a rather inconspicuous part of the hippocampal region; however, it is of major functional importance for several reasons. First, the presubiculum is the only part of the hippocampal formation in which "head direction" cells have been characterized (Taube et al., 1990; Muller et al., 1996). Second, the presubiculum is characterized by input/output relations that are largely different from all other components of the hippocampal region. It receives afferents from retrosplenial and visual cortices, the anterior complex, and the laterodorsal nucleus of the thalamus, and from the claustrum (Swanson and Cowan, 1977; Vogt and Miller, 1983; Witter et al., 1989; Wouterlood et al., 1990). In turn, it distributes a distinct projection to the medial subdivisions of the medial entorhinal cortex (MEA) (Van Groen and Wyss, 1990a). This projection reaches the ipsi- and contralateral MEA, where it terminates in layers I and III. These layers harbor the dendrites of those neurons, the somata of which are located in layers II and III, and which originate the perforant pathway. Neurons in layer II project to the dentate gyrus and CA3, whereas

\footnotetext{
Received Aug. 6, 1996; revised Oct. 31, 1996; accepted Nov. 5, 1996.

This study was supported by a grant from the Human Frontier Science Program Organization. We are much indebted to the late Dr. Hartmut Petter (University of Leipzig) and to Drs. I. Virtanen (University of Helsinki) and R. Buijs (Netherlands Institute for Brain Research) for their generous gifts of antisera to GABA. We thank Peter Goede and Annaatje Pattiselanno for their skillful technical assistance. Dirk de Jong and Shimon Paniry are gratefully acknowledged for photographic processing; Solange van der Linden and Jacqueline van Denderen are acknowledged for critically reading this manuscript.

Correspondence should be addressed to Dr. Theo van Haeften, Department of Anatomy and Embryology, Faculty of Medicine, Vrije Universiteit Amsterdam, Van der Boechorststraat 7, NL-1081 BT Amsterdam, The Netherlands.

Copyright (C) 1997 Society for Neuroscience 0270-6474/97/170862-13\$05.00/0
}

neurons in layer III originate projections to CA1 and the subiculum (Steward, 1976; Steward and Scoville, 1976; cf. Amaral and Witter, 1995). Caballero-Bleda and Witter (1994) have shown that dendrites of principal neurons in layer III of MEA are covered with presubicular appositions, and hence are likely targets for these presubicular inputs.

Principal neurons in layer II of MEA are under powerful inhibitory control, mediated among others by GABAergic basket neurons (Finch et al., 1988; Buhl and Jones, 1993; Jones, 1993; Wouterlood et al., 1995a). Although layer III shows similar numbers of basket neurons (Wouterlood et al., 1995a), the overall inhibition in this layer is low (Jones, 1993; Jones and Buhl, 1993; Van der Linden et al., 1996). Moreover, neurons in layer III of MEA are more vulnerable to epileptic seizures than layer II cells (Du et al., 1993). We have recently proposed that inputs from the presubiculum may be of relevance for these functional differences between neurons in layers II and III (Eid et al., 1996).

In a series of studies, we established that subicular fibers distributing to layers III and V of the entorhinal cortex establish both asymmetrical and symmetrical synapses with entorhinal neurons (Van Haeften et al., 1995a). To substantiate the presence of a possible subicular inhibitory input to MEA, we combined retrograde filling of these projection neurons with immunocytochemistry for GABA. In these experiments, we noted that many GABAergic neurons in the presubiculum (cf. Köhler et al., 1985) were retrogradely labeled. In this study, we have investigated whether these presubicular GABAergic neurons participate in the projections to MEA. Moreover, we investigated whether neurons in the presubiculum give rise to collateral projections to ipsi- and 
contralateral MEA. Finally, presubicular terminals in layers I and III of MEA were morphologically characterized by anterograde tracing, and their postsynaptic targets were identified at the electron microscopical level.

Preliminary reports of this study have been published previously (Van Haeften et al., 1995b; Wouterlood et al., 1995b).

\section{MATERIALS AND METHODS}

In total, 28 female Wistar rats (body weight 200-220 gm; Harlan Centraal Proefdierbedrijf Zeist, The Netherlands) were used: 12 animals for retrograde tracing, combined with GABA immunocytochemistry, and 8 animals for double-fluorescence retrograde tracing. Eight animals were used for anterograde tracing studies and subsequent electron microscopy.

Retrograde tracing and GABA immunocytochemistry. GABAergic neurons in the presubiculum, projecting to MEA, were identified by means of a combination of retrograde tracing and subsequent GABA immunocytochemistry.

Rats were deeply anesthetized with a mixture of ketamine and xylazine (4:3; $10 \%$ solution of Ketaset, Aesco, Boxtel, The Netherlands, and a 2\% solution of Rompun, Bayer, Brussels, Belgium; total dose: $1 \mathrm{ml} / \mathrm{kg}$ body weight) and mounted in a stereotaxic frame. Small holes were made in the skull and $0.4-0.6 \mu \mathrm{l}$ of either a $25 \%$ or a $50 \%$ solution of horseradish peroxidase (HRP; Grade I, Boehringer Mannheim, Mannheim, Germany) in physiological saline was unilaterally injected with a Hamilton syringe. Injections were made under stereotaxical guidance (Paxinos and Watson, 1986) into the superficial layers of either dorsal or ventral parts of MEA.

After a survival time of 7 days, the animals were deeply anesthetized with sodium pentobarbital (Nembutal i.p. $60 \mathrm{mg} / \mathrm{kg}$ body weight, Ceva, Paris, France) and rapidly transcardially perfused with a small volume of physiological saline, followed by $500 \mathrm{ml}$ of a solution of $0.5 \%$ freshly depolymerized paraformaldehyde and 5\% glutaraldehyde (Merck, Darmstadt, Germany) in $0.1 \mathrm{M} \mathrm{NaH} \mathrm{PO}_{4} / \mathrm{Na}_{2} \mathrm{HPO}_{4}$ (phosphate) buffer, $\mathrm{pH}$ 7.4. In case the monoclonal antiserum against GABA was to be used (see below), the brain was perfused with a solution of $4 \%$ paraformaldehyde and $2 \%$ glutaraldehyde in $0.1 \mathrm{~m}$ phosphate buffer, $\mathrm{pH}$ 7.4. After removal from the skull, the brains were post-fixed for $2 \mathrm{hr}$ in the perfusion fixative. Brains were sectioned either on a freezing microtome or with the use of a Vibratome (Technical Products International, St. Louis, MO). In case of freeze-sectioning, brains were cryoprotected by infiltration for $24 \mathrm{hr}$ in a solution of $20 \%$ glycerine and $2 \%$ dimethyl sulfoxide (Merck) in phosphate buffer. Next, brains were frozen onto the stage of a sliding microtome with a solution of $30 \%$ sucrose in phosphate buffer and cut into $40-\mu \mathrm{m}$-thick horizontal or coronal sections. Vibratome sections were cut at $50 \mu \mathrm{m}$ in the horizontal plane. After several rinses in phosphate buffer, the transported HRP was visualized by incubating the sections in nickel-enhanced diaminobenzidine (DAB) substrate: $11 \mathrm{mg}$ nickel ammonium sulfate (Merck) and $25.5 \mathrm{mg}$ of 3,3'-DAB tetrahydrochloride (Sigma, St. Louis, MO), and $6.7 \mu \mathrm{l}$ of a $30 \%$ solution of $\mathrm{H}_{2} \mathrm{O}_{2}$ in $25 \mathrm{ml}$ of $0.1 \mathrm{M}$ phosphate buffer, $\mathrm{pH}$ 7.4. The progress of the histochemical reaction was monitored by inspecting sections at regular time intervals with a light microscope to prevent excessive staining of the HRP-labeled cells. When sufficient staining was achieved, usually after $25 \mathrm{~min}$, the reaction was terminated by rinsing the sections in phosphate buffer. This histochemical reaction produced a black granular precipitate in HRPlabeled neurons. After several rinses in Tris/HCl buffer (Merck), $\mathrm{pH} 7.4$, supplemented with $0.15 \mathrm{M} \mathrm{NaCl}$ [Tris-buffered saline (TBS)], followed by several rinses in TBS containing $0.5 \%$ Triton X-100 (Merck) (TBS-T), the sections were immunocytochemically stained for the presence of GABA. Immunocytochemistry for GABA was carried out with various antisera raised against GABA (Table 1) according to two immunocytochemical protocols. (1) Sections were incubated with the monoclonal antiserum under continuous agitation for $96 \mathrm{hr}$ at $4^{\circ} \mathrm{C}$. Next, the sections were rinsed in TBS-T and incubated in biotinylated horse anti-mouse antiserum (Sigma), diluted 1:100 in TBS-T, for $48 \mathrm{hr}$ at $4^{\circ} \mathrm{C}$. After several rinses in TBS-T, the sections were incubated in avidin-biotin-peroxidase complex (Vectastain, Vector Laboratories, Burlingame, CA) in TBS-T for $18 \mathrm{hr}$ at $4^{\circ} \mathrm{C}$. After rinsing in TBS-T and TBS, followed by several rinses in Tris/ $\mathrm{HCl}, \mathrm{pH} 7.6$, the immunopositive neurons were visualized by reacting the tissue with $10 \mathrm{ml}$ of $0.05 \mathrm{M}$ Tris/ $\mathrm{HCl}, \mathrm{pH} 7.6$, containing $5 \mathrm{mg}$ of DAB and $3.3 \mu \mathrm{l}$ of a $30 \%$ solution of $\mathrm{H}_{2} \mathrm{O}_{2}$ per $10 \mathrm{ml}$. The staining reaction was monitored by viewing sections at regular time intervals, and after sufficient staining (generally after $20 \mathrm{~min}$ ) the reaction was terminated by several rinses in Tris/HCl. (2) Sections reacted with the polyclonal antisera were incubated for $96 \mathrm{hr}$ at $4^{\circ} \mathrm{C}$ and, after several rinses in TBS-T, incubated with swine anti-rabbit IgG (Dakopatts, Copenhagen, Denmark) and diluted 1:100 in TBS-T for $18 \mathrm{hr}$ at room temperature. After several rinses in TBS-T, the sections were subsequently incubated in rabbit peroxidase-antiperoxidase (Dakopatts), diluted 1:200 in TBS-T for $3 \mathrm{hr}$ at room temperature. The staining reaction was visualized with DAB as described above.

All sections were mounted on glass slides from a solution of $0.1 \%$ gelatin in Tris/ $\mathrm{HCl}, \mathrm{pH} 7.6$, air-dried, dehydrated, cleared in xylene, and coverslipped with Entellan (Merck). The location and number of GABAergic neurons and HRP-containing neurons, as well as doublelabeled neurons, were determined using MDplot plotting software (Minnesota Datametrics, St. Paul, MN).

Double-fluorescence retrograde tracing. The degree of collateralization of presubicular projections to the ipsi- and contralateral MEA was determined by retrograde transport of two different fluorescent tracers according to an experimental protocol well established in our laboratory (for a methodological discussion, see Dolleman-van-der-Weel and Witter, 1996).

Glass micropipettes (GC-150F-15, Clark, Reading, UK) with a tip diameter of $10-15 \mu \mathrm{m}$ were filled with either a $1.25 \%$ solution of Fast Blue (FB; Dr. Illing, Frankfurt, Germany) in 0.1 M sodium cacodylate (Merck), $\mathrm{pH} 7.4$, or with a $2 \%$ solution of diamidino yellow (DY; Dr. Illing) in $0.1 \mathrm{M}$ phosphate buffer, $\mathrm{pH}$ 7.4. All injections were made unilaterally in layers I-III of dorsal MEA. FB was injected in one side of MEA by applying positive-pulsed DC currents $(4-5 \mu \mathrm{A}, 7 \mathrm{sec}$ on, $7 \mathrm{sec}$ off) for $5 \mathrm{~min}$, whereas small amounts of DY $(0.2-0.4 \mu \mathrm{l})$ were injected in the contralateral MEA by applying pressure pulses over a period of 20 min. After 2 weeks of survival, the animals were sacrificed and perfused

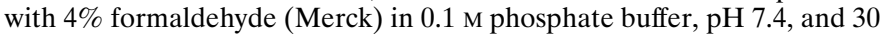
$\mu \mathrm{m}$ sections were cut in the horizontal plane on a freezing microtome. Sections were mounted from a $0.1 \%$ gelatin solution onto slides, airdried, and stored at $-40^{\circ} \mathrm{C}$ until further use. For analysis, slices were thawed and examined with the use of a fluorescence microscope (Zeiss ACM IV F, equipped with filter mirror system 01, $365 \mathrm{~nm}$ ), and the positions of labeled neurons were plotted using MDplot plotting software. After plotting, the sections were stained in a $0.2 \%$ aqueous solution of cresyl violet (Merck), dehydrated, and coverslipped. The exact location of the labeled cells in the presubiculum was verified by projecting the contours and cell layers of the presubiculum, as revealed by the cresyl violet staining, onto the plots.

Anterograde tracing and electron microscopy. The morphology of presubicular terminals in the superficial layers of MEA was revealed by means of anterograde tracing of the presubicular projections and subsequent analysis of the labeled terminals at the ultrastructural level.

Glass micropipettes (GC-150F-15, Clark) with a tip diameter of 10-15 $\mu \mathrm{m}$ were filled with a $5 \%$ solution of $10 \mathrm{kDa}$ biotinylated dextran amine

Table 1. GABA antisera, fixatives, and dilutions used for GABA immunocytochemistry

\begin{tabular}{lllll} 
Antiserum & Source & Fixative & Dilution & Reference \\
\hline $\begin{array}{l}\text { Rabbit anti-GABA } \\
\text { "Moortje" }\end{array}$ & Netherlands Institute for Brain Research & $0.5 \%$ formaldehyde & $1: 1000$ & Buijs et al. (1987) \\
Rabbit anti-GABA & (Amsterdam, The Netherlands) & $5 \%$ glutaraldehyde & & \\
& Dr. H. Petter (Leipzig, Germany) & $0.5 \%$ formaldehyde & $1: 4000$ & - \\
$\begin{array}{l}\text { Mouse anti-GABA } \\
\text { (monoclonal) }\end{array}$ & Dr. I. Virtanen (Helsinki, Finland) & $\begin{array}{l}5 \% \text { glutaraldehyde } \\
4 \% \text { formaldehyde }\end{array}$ & $1: 500$ & Szabat et al. (1992) \\
\hline
\end{tabular}




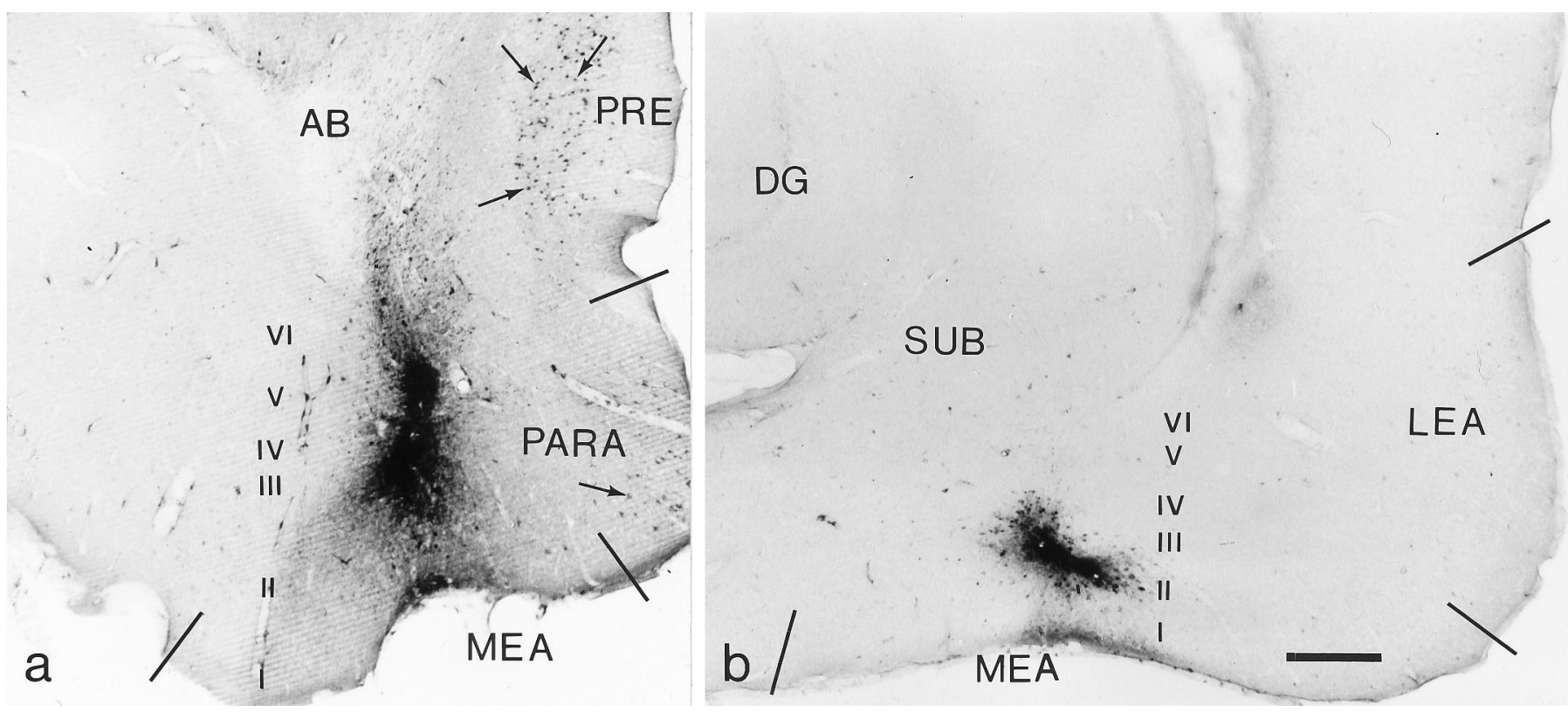

Figure 1. Photomicrographs of $40 \mu \mathrm{m}$ horizontal sections showing representative examples of HRP injection sites and their dimensions in the $M E A$. $a$, Vibratome section showing the main focus of an HRP injection in the deep and superficial layers of dorsal MEA. Arrows point to retrogradely labeled neurons in the dorsal presubiculum (PRE) and parasubiculum (PARA). Roman numerals indicate the layers of MEA. Lines indicate the boundaries of $M E A$, parasubiculum, and presubiculum. $A B$, angular bundle. $b$, Frozen section showing the ventral $M E A$ with an HRP injection site located mainly in the superficial layers. Roman numerals indicate the layers of MEA. Lines indicate the boundaries of the lateral entorhinal cortex (LEA) and $M E A$. SUB, Subiculum; $D G$, dentate gyrus. Scale bar, $250 \mu \mathrm{m}$.

(BDA; Molecular Probes, Eugene, OR) in $0.01 \mathrm{~m}$ phosphate buffer, $\mathrm{pH}$ 7.3. Unilateral injections were stereotaxically placed into either the ventral or dorsal presubiculum by lowering the pipette tip into the desired coordinates and applying positive-pulsed DC currents $(6.5 \mu \mathrm{A}, 7 \mathrm{sec}$ on, $7 \mathrm{sec}$ off) for $10 \mathrm{~min}$. After 1 week of survival, the animals were sacrificed by perfusion with a solution of $4 \%$ depolymerized paraformaldehyde,
$0.05 \%$ glutaraldehyde, and $0.2 \%$ picric acid (Merck) in $0.125 \mathrm{M}$ phosphate buffer, $\mathrm{pH}$ 7.4. After removal from the skull, $50-\mu \mathrm{m}$-thick coronal brain sections were cut with a vibratome. After cryoprotection, as described above, sections were subjected to several cycles of freeze thawing (for details, see Wouterlood et al., 1993). All subsequent rinses and incubations took place in TBS, $\mathrm{pH}$ 7.6. Sections were incubated for $14 \mathrm{hr}$ in

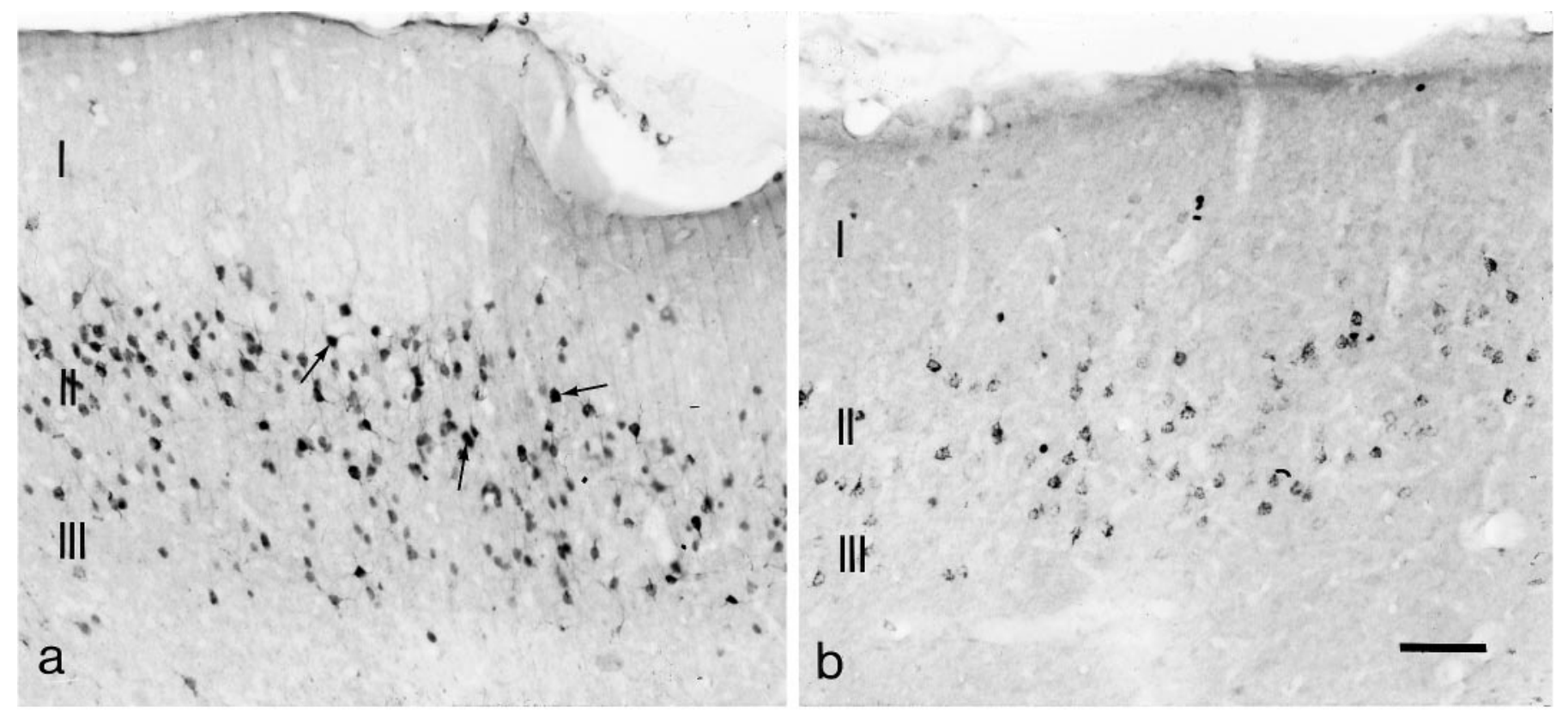

Figure 2. Photomicrographs of $40 \mu \mathrm{m}$ horizontal sections showing the distribution of retrogradely HRP-labeled neurons in the dorsal presubiculum after a unilateral injection in the dorsal MEA. $a$, Retrogradely labeled neurons in the dorsal presubiculum ipsilateral of the injection site. Arrows indicate neurons that are completely filled with HRP. Note the absence of retrogradely labeled neurons in layer I. Roman numerals indicate the different layers of the presubiculum. $b$, Contralateral dorsal presubiculum at the same dorsoventral level as in $a$. Note that the number of retrogradely labeled neurons is less when compared with the number of labeled neurons in the ipsilateral presubiculum, and that completely filled neurons are almost absent. Roman numerals indicate the layers of the presubiculum. Scale bar, $75 \mu \mathrm{m}$. 


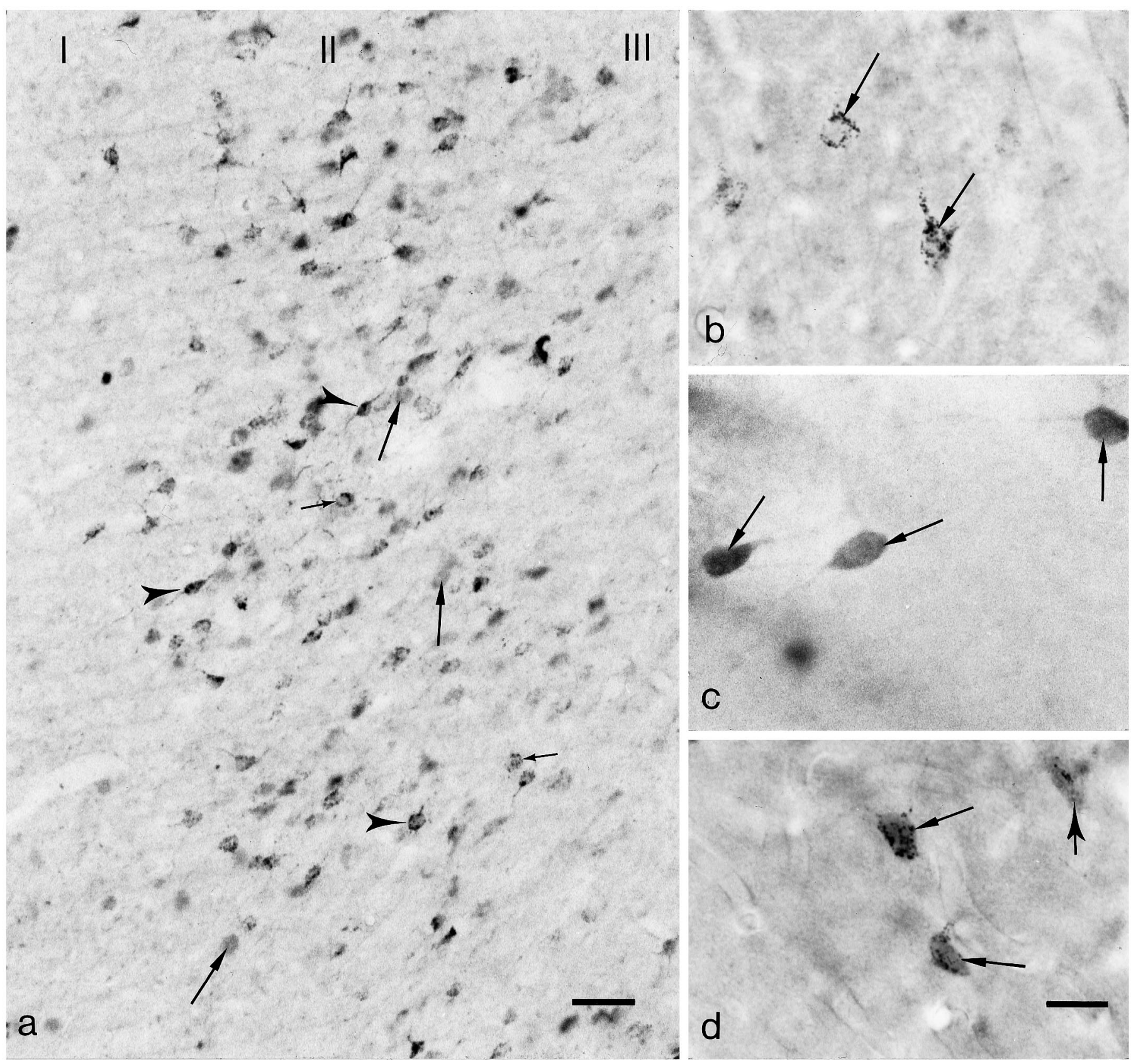

Figure 3. Photomicrographs of horizontal $40 \mu \mathrm{m}$ sections of the dorsal presubiculum showing single- and double-labeled neurons. $a$, The ipsilateral presubiculum, taken from a brain in which HRP was injected in the dorsal MEA, after staining for both HRP and GABA. Many retrogradely labeled neurons (small arrows), GABA-containing neurons (large arrows), and neurons containing both GABA and HRP (arrowheads) are present in layers II and III. Roman numerals indicate the superficial layers of the presubiculum. Scale bar, $50 \mu \mathrm{m}$. $b$, Neurons in layer II of the ipsilateral dorsal presubiculum stained for retrogradely transported HRP. Note the typical granular inclusions in the cytoplasm (arrows). c, GABAergic neurons (arrows) in layer II of the ipsilateral dorsal presubiculum. $d$, Double-labeled neurons in layer II of the ipsilateral dorsal presubiculum containing both GABA and retrogradely transported HRP (arrows). A retrogradely HRP-labeled neuron is indicated by a large vertical arrow. Scale bar, $20 \mu \mathrm{m}$ in $b-d$.

avidin-biotin-peroxidase complex (overnight at $4^{\circ} \mathrm{C}$ ), rinsed in Tris/ $\mathrm{HCl}$, $\mathrm{pH} 8.0$, and incubated in $\mathrm{DAB}$, as described for the visualization of GABA immunoreactivity. When sufficient DAB precipitate had been formed, the reaction was terminated by rinsing the sections in Tris/ $\mathrm{HCl}$. Post-fixation took place for $1 \mathrm{hr}$ in cold $1 \% \mathrm{OsO}_{4}$ in $0.1 \mathrm{~m}$ sodium cacodylate buffer (Merck), $\mathrm{pH}$ 7.4. Next, the sections were stained in an ice-cold $2 \%$ aqueous uranyl acetate solution (Merck), dehydrated through ascending series of alcohol and, through two rinses of propylene oxide, flat-embedded in Epon (Polysciences, Warrington, PA) between polyethylene foils. After curing $\left(24 \mathrm{hr}\right.$ at $\left.50^{\circ} \mathrm{C}\right)$, all sections were transferred to slides, and the position of the BDA-labeled fiber plexus was determined under the light microscope. Brain slices with a high plexus density in layers I and III of MEA were selected, photographed, and drawn using a microscope equipped with a camera and a camera lucida system. Sample areas were removed from these layers with a scalpel and glued onto precured Epon resin blocks. Continuous series of ultrathin sections were cut on a Reichert OM-U4 ultramicrotome. The series of sections were mounted on Formvar-coated slot grids, contrasted with lead citrate, and examined in a Philips EM-301 electron microscope.

Specificity controls. Specificity of the immunoreaction for GABA was determined by incubating the sections in the absence of the primary antisera, i.e., TBS-T only for $96 \mathrm{hr}$ at $4^{\circ} \mathrm{C}$. After several rinses in TBS-T, the sections were further processed as described above. To have a control for the possible presence of residual unstained HRP after DAB-nickel 


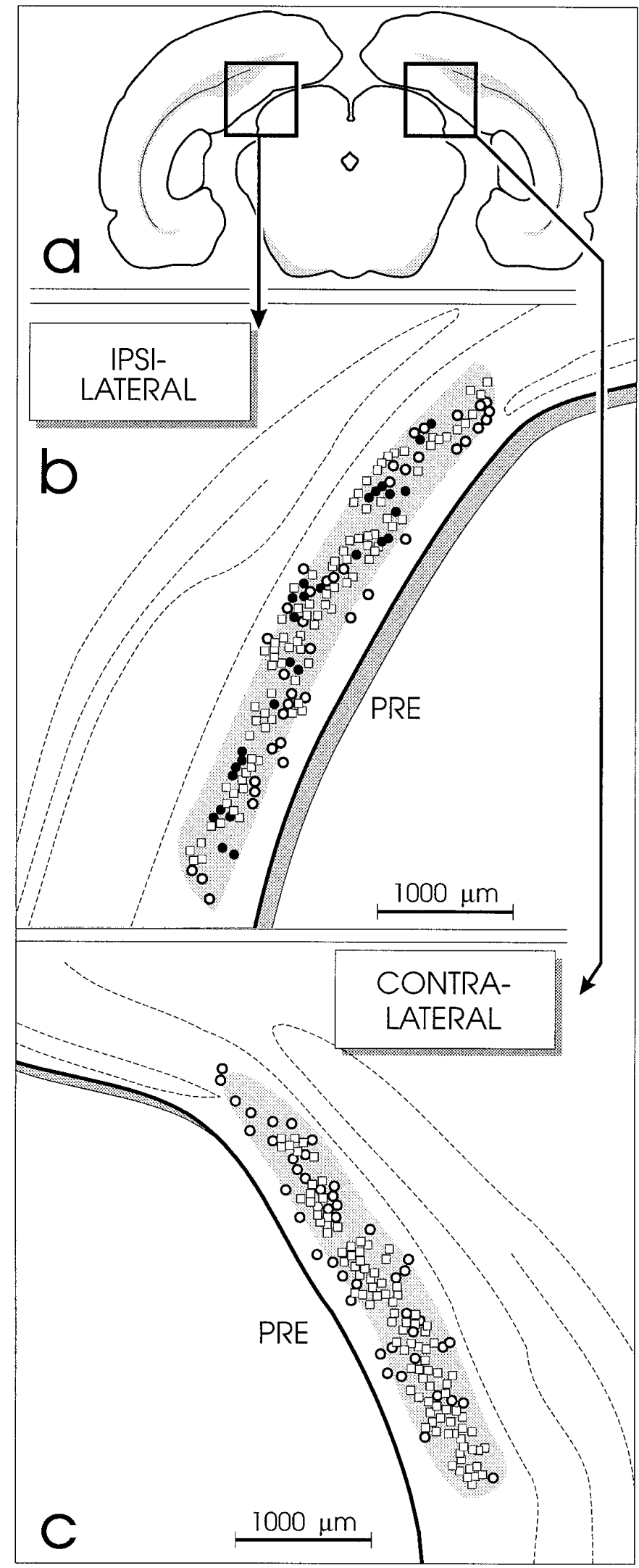

Figure 4. Schematic representation of the distribution of GABAimmunopositive, HRP-labeled, and double-labeled neurons in the ipsilateral and contralateral dorsal presubiculum after a unilateral HRP injection in the dorsal MEA and subsequent GABA immunocytochemistry. $a$, Representation of the position of the dorsal presubiculum as seen in staining, randomly selected sections were first incubated in nickelenhanced $\mathrm{DAB}$ substrate, immediately followed by an incubation in common DAB substrate. With this procedure, the presence of residual HRP in sections would result in a brown staining of structures.

\section{RESULTS}

\section{Specificity controls}

In those cases in which the primary antisera against GABA had been substituted by TBS-T, the subsequent immunocytochemical procedure never resulted in staining of neurons in any part of the brain. This indicates that the results of our experiments are not attributable to aspecific binding of secondary and tertiary antibodies to tissue antigens. For more details on the specificity of the GABA-antisera used, we refer to Szabat et al. (1992) and to Buijs et al. (1987) (see Table 1).

Sections, taken from brains in which HRP had been deposited, were treated with nickel-enhanced DAB substrate and subsequently incubated in common $\mathrm{DAB}$ substrate. This procedure never resulted in brown-stained cells or other structures. This demonstrated that no residual unstained HRP was present in the sections after DAB-nickel staining of HRP.

\section{Retrograde tracing and GABA immunocytochemistry}

For this study, we analyzed six cases with injections in the dorsal part of MEA and six cases that received injections in the ventral part of MEA. In the dorsal MEA, generally both deep and superficial layers were involved in the injection site (Fig. 1a). In contrast, in the ventral part of MEA, injections were generally restricted to the superficial layers (Fig. 1b). Notwithstanding these differences in laminar involvement, injections in both parts of MEA resulted in strikingly similar patterns of labeling in the presubiculum. Marked retrograde labeling of neurons was seen in layers II and III of both the ipsi- (Fig. 2a) and contralateral presubiculum (Fig. 2b). No retrogradely labeled neurons were present in layer I and the deeper layers of the presubiculum. The retrograde labeling of presubicular neurons appeared to be organized according to a ventral-to-dorsal topography; i.e., injections in ventral parts of MEA resulted in retrograde labeling of neurons in ventral parts of the presubiculum, whereas injections in dorsal parts of MEA resulted in labeling of neurons in dorsal parts of the presubiculum.

Immunocytochemical staining with antibodies against GABA revealed the presence of GABAergic neurons in the superficial layers and in the deep layers of both ipsi- and contralateral ventral and dorsal presubiculum. Incidentally, a few positive neurons could be observed in layer I. All three antisera stained neurons with high selectivity, and no major differences in both location and the number of stained neurons were observed. Under all experimental circumstances, however, the monoclonal antiserum yielded the most optimal staining of GABAergic neurons. This antibody appeared to stain somata, and to some extent, also dendrites, whereas the polyclonal antibodies mostly stained somata only. Moreover, the monoclonal antiserum produced only a very low background staining of the surrounding neuropil. For

$\leftarrow$

coronal sections. The squares depict the areas that are shown enlarged in $b$ and $c$. $b$, Distribution of HRP-labeled (open squares), GABAimmunopositive (open circles), and double-labeled ( filled circles) neurons in the dorsal presubiculum (PRE), ipsilateral to the HRP injection site. $c$, Distribution of HRP-labeled (open squares) and GABA-immunopositive (open circles) neurons in the dorsal presubiculum (PRE), contralateral to the HRP injection site. No double-labeled neurons are present. 
these reasons, most results presented here are derived from cases using the monoclonal antibody.

After unilateral HRP injection in dorsal MEA, colocalization of HRP and GABA in presubicular neurons appeared to be restricted to neurons located in the ipsilateral dorsal presubiculum. (Figs. 3, 4a,b). In all cases, no colocalization of HRP and GABA occurred in neurons located in the contralateral dorsal presubiculum (Fig. 4a,c). In those cases in which HRP injections had been made in the ventral MEA, no colocalization was observed in neurons in the ipsilateral or in the contralateral ventral presubiculum.

In each case, cell counts were performed on at least two series of 16 sections containing the entire presubiculum. It appeared that $30-40 \%$ (mean of 6 cases) of all GABAergic neurons in the ipsilateral dorsal presubiculum, as revealed by immunocytochemistry, were double-labeled (Fig. 4a,b).

\section{Double-fluorescence retrograde tracing}

The above findings indicate that GABAergic neurons in the dorsal presubiculum project to ipsilateral MEA only. In contrast, nonGABAergic-labeled neurons seem to innervate both the ipsi- and contralateral MEA. It is not known whether it is a general organizational principle of presubicular projections to MEA, that they distribute strictly unilaterally, be it to the ipsilateral or contralateral hemisphere. Alternatively, non-GABAergic presubicular projections may distribute collateralized projections to both the ipsiand contralateral MEA. We investigated this with the use of double-fluorescence retrograde tracing techniques.

In eight animals, injections with DY and FB were successfully placed such that both the left and the right MEA were injected with one of the two tracers. As a result of these unilateral injections, FB and DY fluorescent neurons were found to be present in layers II and III of both ipsi- and contralateral presubiculum. The distribution of neurons as revealed by the retrograde tracers did not significantly differ between the ipsi-and contralateral hemisphere. Careful examination of the retrogradely labeled fluorescent neurons in the superficial layers of the presubiculum showed that the two populations of neurons overlap. In all cases, however, no double-labeled neurons were observed. This finding demonstrates that ipsi- and contralateral projections to MEA originate from different populations of presubicular neurons (Fig. 5).

\section{Anterograde tracing and electron microscopy}

It is generally believed that GABAergic axon terminals form symmetrical synapses with their targets (Ribak, 1992; Soriano and Frotscher, 1993; Pickel and Chan, 1995). To further substantiate the light microscopical finding of a strictly ipsilateral GABAergic presubicular projection to MEA, we carried out an electron microscopical analysis. This series of experiments also supplied information concerning whether the postsynaptic targets of presubicular fibers that form symmetrical synapses are different from those forming asymmetrical synapses. Unilateral injections with the anterograde tracer BDA in the presubiculum resulted in a massive staining of fibers terminating in layers I and III of both the ipsi- and contralateral MEA (Fig. $6 a, b$ ). In layers II and Va, only a few fibers were stained. No distinction could be made in the pattern of termination of fibers originating from neurons in the dorsal and ventral presubiculum. In all cases, the pattern of labeling in contralateral MEA was the mirror image of that present in ipsilateral MEA.

Electron microscopical analysis of 200 randomly sampled, serially sectioned terminals in layers I and III of ipsilateral dorsal MEA revealed the presence of many BDA-positive terminals forming "classical" asymmetrical (85\% of total number sampled) synapses with entorhinal neurons. These synapses were characterized by the presence of a thick postsynaptic density and a wide synaptic cleft (cf. Gray, 1959; Colonnier, 1968; Peters et al., 1991) (Fig. 7a,b). Among the targets of the labeled asymmetrical synapses, we observed both dendritic shafts (Fig. 7a) and spines (Fig. 7b) in an almost equal percentage. A small percentage $(15 \%)$ of the terminals, however, formed synapses that displayed features characteristic of symmetrical synapses, i.e., a thin postsynaptic density and a small translucent synaptic cleft (Fig. 7c) (cf. Gray, 1959; Colonnier, 1968; Peters et al., 1991). Symmetrical synapses had dendritic shafts as postsynaptic element only (Fig. 7c). Layers I and III did not differ in their ratios of both synapse types. Serial reconstruction of dendritic shafts showed that many of them did not bear spines and received converging unlabeled synapses (Fig. 8).

In the contralateral dorsal MEA, BDA-labeled terminals did not form symmetrical synapses in layers I and III. Here, all BDA-labeled terminals $(n=80)$ appeared to form synapses of the asymmetrical type on both spines and dendritic shafts in an equal percentage.

In the ventral MEA, both in the ipsilateral and contralateral hemisphere, random sampling of BDA-positive terminals $(n=$ 100) present in the terminal field in layers I and III did not reveal the presence of any symmetrical synapses. All synapses were of the asymmetrical type terminating on both spines and dendritic shafts in an almost equal ratio.

\section{DISCUSSION}

The present findings in the rat confirm previous reports that presubicular afferents to the entorhinal cortex arise predominantly from neurons located in the superficial layers of the presubiculum (Segal, 1977; Beckstead, 1978; Eid et al., 1996). These projections terminate bilaterally in MEA with a dense plexus in layers I and III, and to a lesser extent in layers II and Va. Moreover, these projections are topographically organized such that dorsal parts of the presubiculum project to dorsal parts of MEA, whereas fibers originating from ventral portions of the presubiculum predominantly reach the ventral parts of MEA (Köhler et al., 1978; Köhler, 1984, 1985; Van Groen and Wyss, 1990a; Caballero-Bleda and Witter, 1993, 1994).

Although the total number of GABAergic and retrogradely HRP-labeled neurons in the presubiculum varied between rats because of differences in GABA fixation and tracer deposit and transport, the proportion of double-labeled neurons appeared to be rather similar in each case. Our main finding is that a substantial number of GABAergic neurons in the presubiculum contribute to projections to MEA (Figs. $4 b, 9$ ). These GABAergic projections only distribute to the ipsilateral MEA. No GABAergic projections to the contralateral MEA were found (Figs. 4c, 9). Moreover, our results indicate that this GABAergic component originates only from the dorsal presubiculum and, in line with the overall topography, distributes to the dorsal part of MEA. No GABAergic projections were found that originate in the ventral presubiculum and reach the ventral part of MEA (Fig. 9). Finally, the results of the double-fluorescent retrograde tracing study show that all presubicular neurons selectively project to either the ipsilateral or the contralateral MEA (Figs. 5, 9). No neurons were found that project to both hemispheres. 


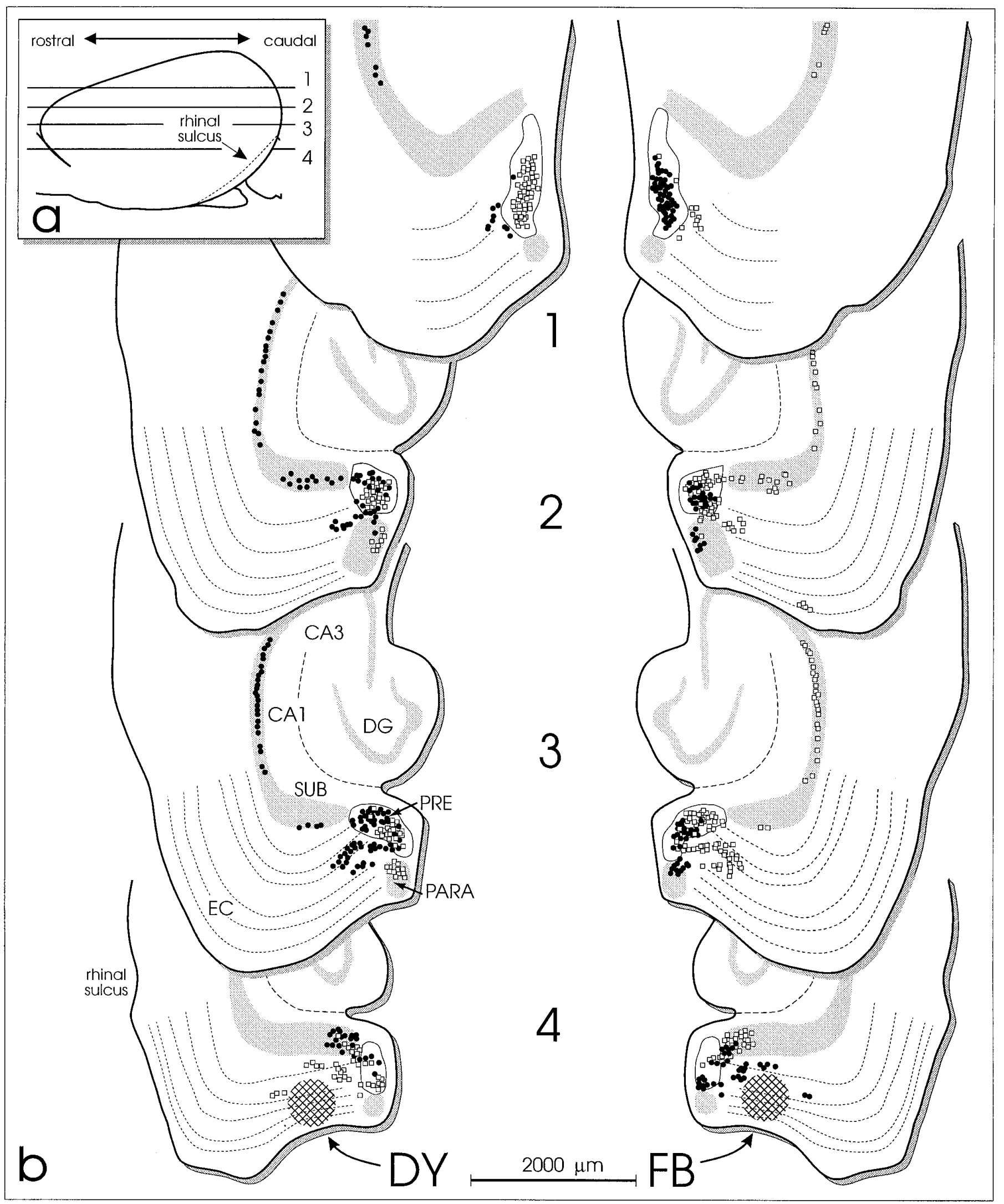

Figure 5. Distribution of retrogradely labeled neurons in the ipsilateral and contralateral presubiculum at four different dorsoventral levels. The rat was injected in the superficial layers of the dorsal entorhinal cortex $(E C)$ with the retrograde tracers FB (right hemisphere) and DY (left hemisphere). a, Schematic lateral view of the brain. Horizontal lines indicate the dorsoventral level of the horizontal sections shown in $b . b$, Schematized camera lucida drawings from Nissl-stained horizontal sections $(30 \mu \mathrm{m})$. Figure legend continues. 


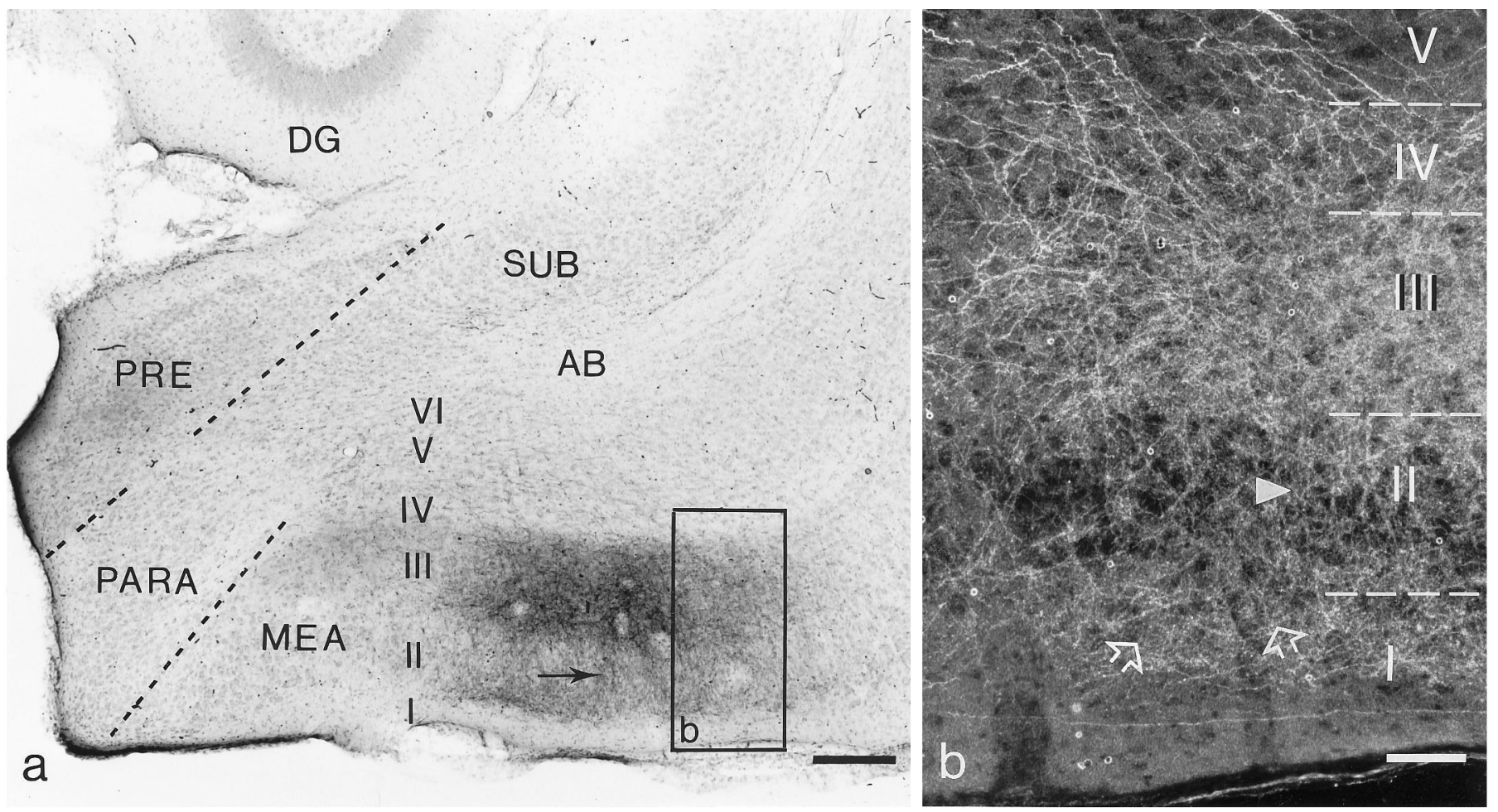

Figure 6. Photomicrographs of horizontal $40 \mu \mathrm{m}$ sections showing anterograde BDA labeling of the presubiculo-entorhinal projection. $a$, Dense plexus of anterogradely labeled fibers is present in the deep part of layer I and in layer III of the MEA. Columns of labeled fibers are present in layer II (arrow). Dashed lines indicate the borders between the subiculum $(S U B)$ and the presubiculum (PRE), between the presubiculum and the parasubiculum (PARA), and between parasubiculum and MEA. The square depicts the area that is shown in more detail in $b$. Roman numerals indicate the layers of MEA. DG, dentate gyrus; $A B$, angular bundle. Scale bar, $200 \mu \mathrm{m}$. $b$, Dark-field photomicrograph showing a detail of the presubicular terminal plexus in layers I and III. Arrows point to presubicular fibers in the deep part of layer I. Arrowhead indicates a column of fibers in layer II. Roman numerals indicate the layers of MEA. Scale bar, $90 \mu \mathrm{m}$.

\section{Characterization of presubicular neurons}

At least four distinct populations of neurons can be distinguished in the presubiculum (Fig. 9): (1) non-GABAergic neurons projecting to ipsilateral MEA; (2) non-GABAergic neurons projecting to contralateral MEA; (3) GABAergic neurons projecting to ipsilateral MEA; and (4) GABAergic interneurons.

\section{Non-GABAergic projection neurons}

Two types of non-GABAergic projection neurons were identified in the presubiculum: those that project to ipsilateral MEA and those projecting to contralateral MEA (Figs. 5, 9). Both types are not restricted to a specific superficial layer of the presubiculum and can be found in both ventral and dorsal parts of the presubiculum.

Although the bilateral projection to MEA has been described in detail in rats (Köhler, 1984, 1985; Van Groen and Wyss, 1990a; Caballero-Bleda and Witter, 1993) and guinea pigs (Shipley, 1975), this is the first report that ipsi- and contralateral projections originate from different subsets of presubicular projection neurons. Interestingly, these two populations of projection neurons are not spatially separated, but overlap within the presubiculum. Nothing is known about the distribution of the inputs to the presubiculum in relation to these two populations of projection neurons. Moreover, we do not know whether the intrinsic network of the presubiculum facilitates integration or separation between these populations. Therefore, the functional significance of this specialization remains unclear.

\section{GABAergic neurons}

GABAergic neurons are distributed throughout the superficial and deep layers of the dorsal and ventral presubiculum. Their overall density and distribution is in line with previous reports (Köhler et al., 1985). In view of our observations that GABAergic neurons project to MEA, but that they do so only in the dorsal presubiculum, we wanted to make sure that such results were not caused by unwanted selectivity or cross-reactivity of the antibody. Therefore, we used several antibodies obtained from various sources (see Table 1), of which the selectivity has been established (Buijs et al., 1987; Szabat et al., 1992). After immunostaining, the antibodies showed no major variance in both the location and the number of GABAergic neurons in the presubiculum. Moreover, the GABA antibodies did not cross-react with the retrogradely labeled neurons. Therefore, we feel confident that our immunocytochemical approach provided us with a reliable estimate of the distribution of GABAergic neurons in the presubiculum.

Two populations of GABAergic neurons were revealed in the

Hatched areas in both hemispheres indicate the spread of each of the tracers at the injection sites. The position of each of the labeled neurons is indicated with a filled circle for neurons retrogradely labeled with FB and with an open square for neurons labeled with DY. Dashed lines indicate the layers of EC. $D G$, Dentate gyrus; $S U B$, subiculum; $C A 1$, hippocampal field CA1; $C A 3$, hippocampal field CA3; PRE, presubiculum; $P A R A$, parasubiculum. 

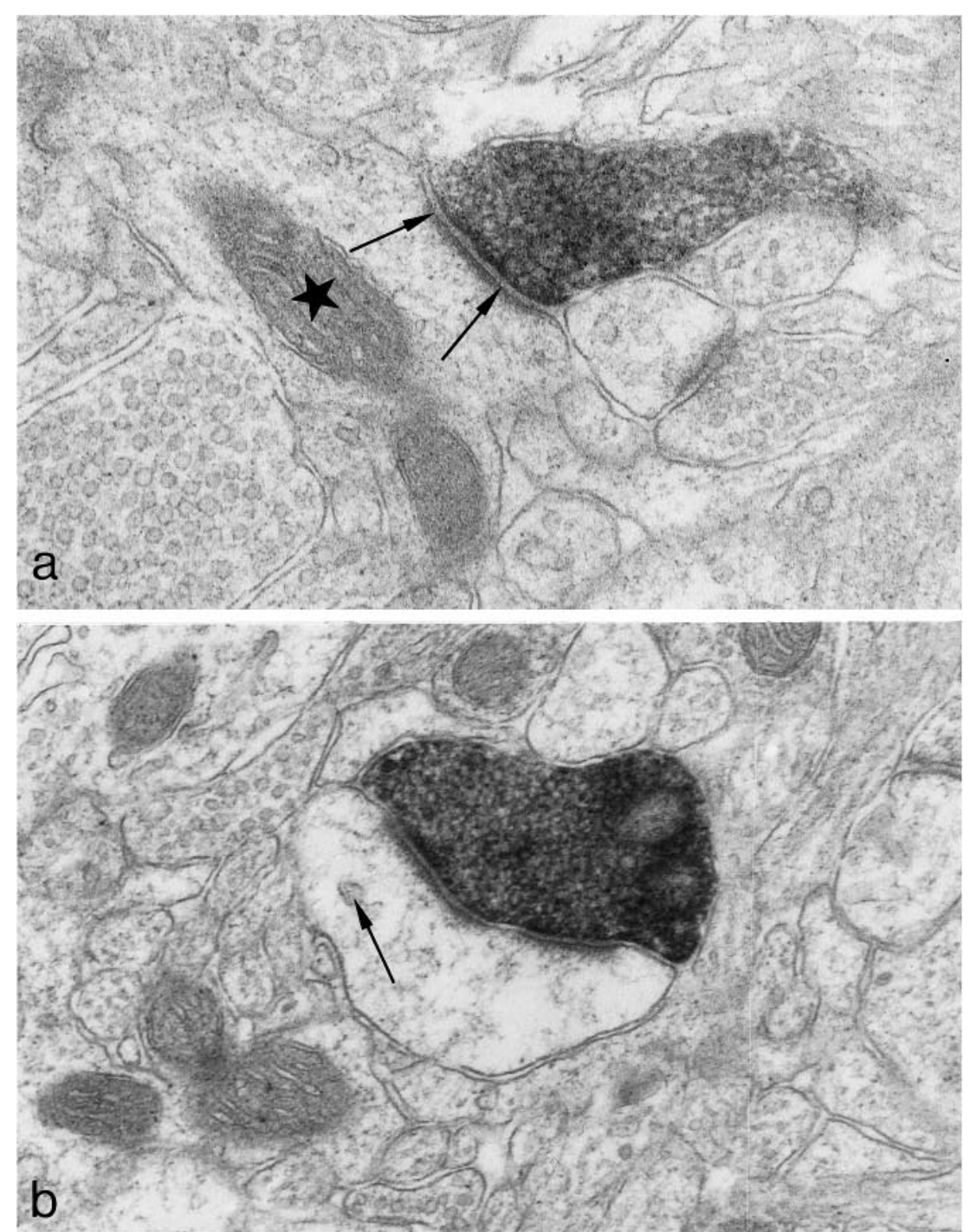

Figure 7. Electron micrographs of BDA-labeled presubicular terminals forming synapses in the superficial layers of the dorsal MEA. $a$, A bouton making an asymmetrical synapse with a dendritic shaft. Arrows point to a well developed postsynaptic density. Note the opaque diaminobenzidine reaction product obscuring the vesicles in the synaptic terminal. Asterisk indicates a mitochondrion in the dendritic cytoplasm. $b$, Asymmetrical synapse on a spine. Arrow points to spine apparatus. $c$, Symmetrical synapse on a dendritic shaft. Large arrows point to microtubuli in the dendritic cytoplasm. Note the translucent synaptic cleft (arrowhead) and the absence of a well developed postsynaptic density (small arrows). Asterisk indicates a mitochondrion in the dendritic cytoplasm. Scale bar, $100 \mathrm{~nm}$.

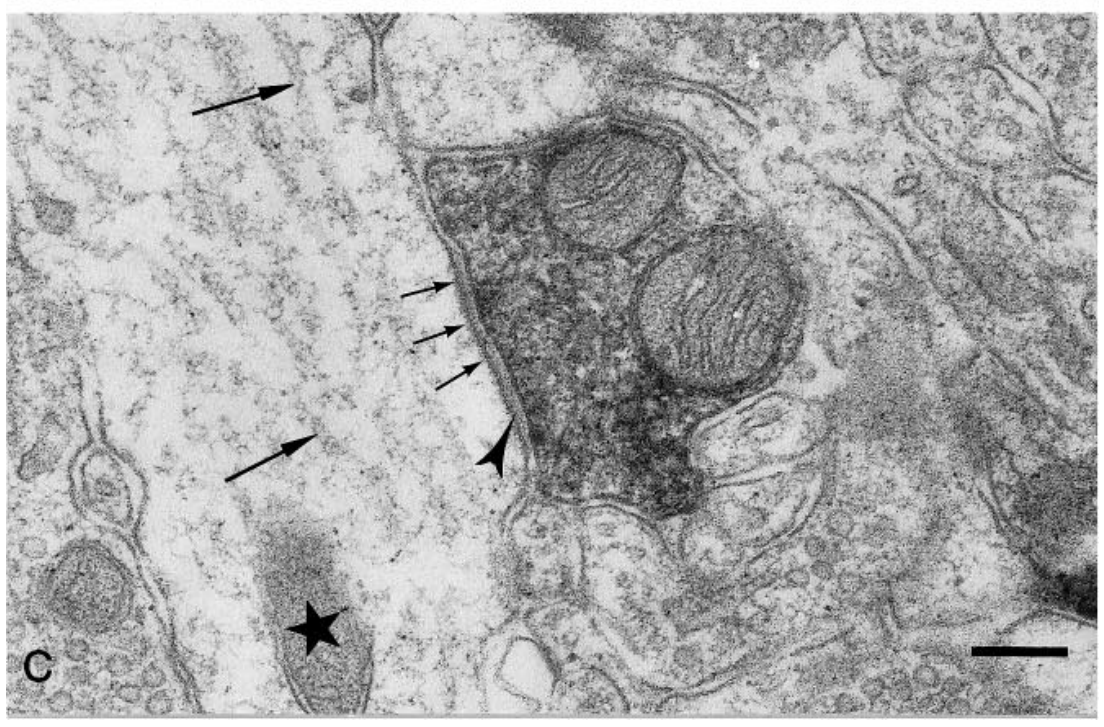

presubiculum (Figs. 4b, 9): (1) GABAergic neurons, the axons of which do not reach the entorhinal cortex, and (2) GABAergic projection neurons of which the axons reach the ipsilateral MEA. The first type of GABAergic neuron is widely distributed through- out the superficial and deep layers of the dorsal and ventral presubiculum. They most likely represent the GABAergic interneurons described by Köhler et al. (1985). The second population, which represents projection neurons, is present only in layers II 

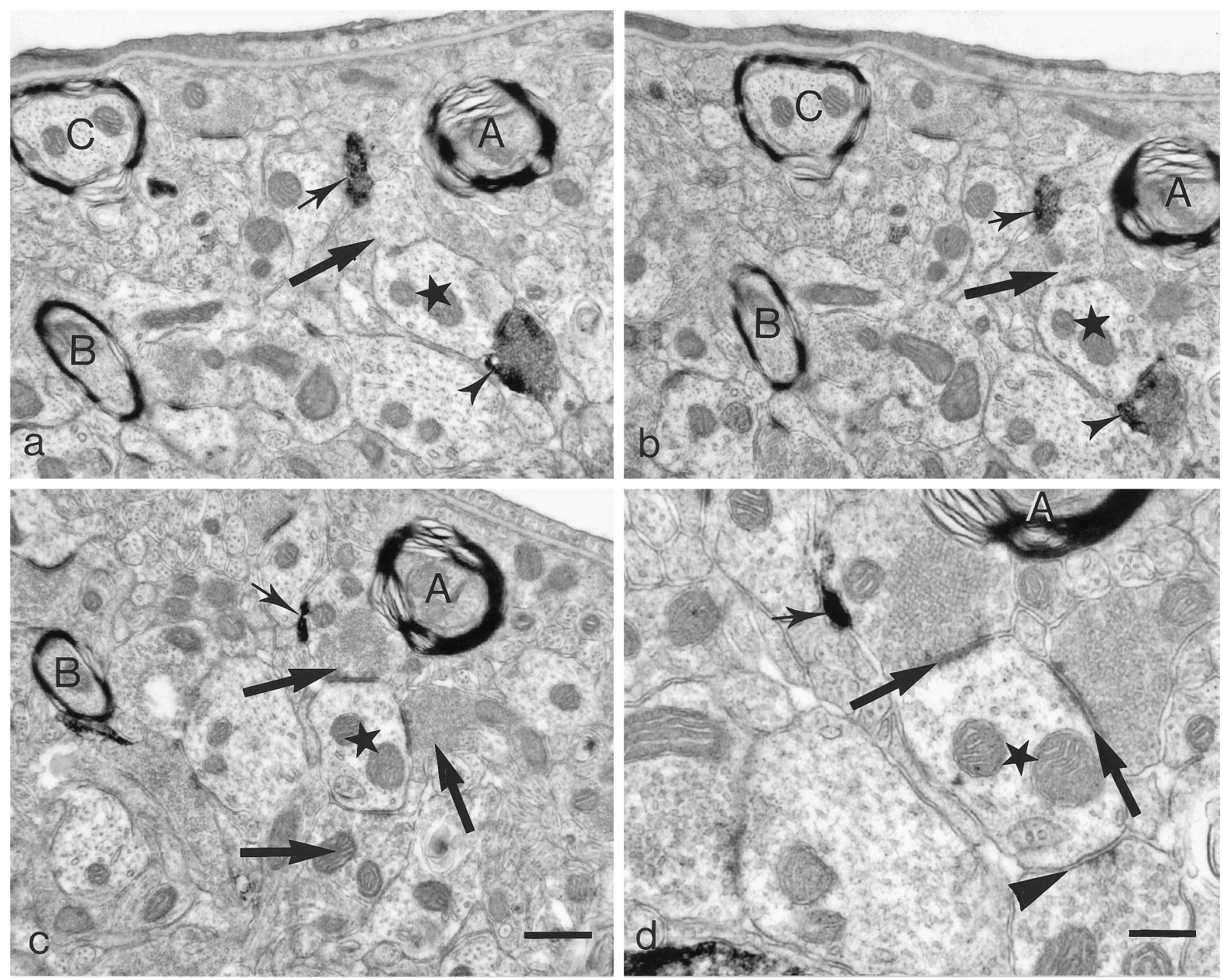

Figure 8. Electron micrographs showing a serial reconstruction of a spine-free dendritic shaft that receives a synaptic contact from a BDA-labeled presubicular terminal. $a$, Asymmetrical BDA-labeled terminal (arrowhead) forming a synapse with a dendritic shaft (asterisk). Note that the same dendritic shaft is receiving a synapse from another unlabeled terminal (thick arrow). Transversely cut axons $(A-C)$ function as landmarks. Small arrow indicates a BDA-labeled structure. $b$, A section at $700 \mathrm{~nm}$ distance from the section shown in $a$. A-C indicate the same transversely cut axons as shown in $a$. Asterisk denotes the dendritic shaft with a labeled terminal (arrowhead) and an unlabeled terminal (thick arrow) forming asymmetrical synapses. Small arrow indicates the same BDA-labeled structure as shown in the previous photomicrograph. $c$, A section at $1400 \mathrm{~nm}$ distance from the section shown in $a$. The letters $A$ and $B$ indicate the landmarks as shown in $a$ and $b$. The dendrite (asterisk) receives three converging synapses (thick arrows). Small arrow denotes the BDA-labeled structure. $d$, Section at $1600 \mathrm{~nm}$ distance from the section as shown in $a$. The letter $A$ denotes one of the axons that functions as a landmark. The dendritic shaft (asterisk) receives two asymmetrical synapses (thick arrows) and a symmetrical synapse (arrowhead). Small arrow points to the BDA-labeled structure. Scale bars: $a-c, 600 \mathrm{~nm} ; d, 300 \mathrm{~nm}$.

and III of the dorsal presubiculum. Although we have shown that the axons of neurons of the second group reach MEA, we cannot exclude that these neurons have a local arborization in the presubiculum or in other parts of the brain as well. That GABAergic presubicular neurons indeed contribute to the projections to MEA is corroborated by our ultrastructural findings. First, we observed that a small fraction of the labeled presubicular terminals in MEA displays features that are characteristic for symmetrical synapses (Fig. 7c) (Gray, 1959; Colonnier, 1968; Peters et al., 1991). It is generally accepted that symmetrical synapses are associated with GABAergic neurons (Ribak, 1992; Soriano and Frotscher, 1993; Pickel and Chan, 1995). Second, labeled terminals forming symmetrical synapses have been found in the dorsal part of ipsilateral MEA only, not in the ventral part or in the entire contralateral MEA. Thus, this distribution of symmetrical synapses is perfectly in line with our data, indicating that GABAergic projection neurons are found only in the ipsilateral dorsal presubiculum. GABAergic projection neurons have been demonstrated previously in the entorhinal cortex (Germroth et al., 1989), septum (Allan and Crawford, 1984; Freund, 1989), and dentate gyrus (Seress and Ribak, 1983).

\section{Targets of presubicular projections}

A majority of the presubicular terminals form asymmetrical synapses with both dendritic shafts and spines of neurons in dorsal and ventral MEA (Fig. 7a,b). Symmetrical synapses have only dendritic shafts of entorhinal neurons in ipsilateral dorsal MEA as postsynaptic elements (Fig. $7 c$ ). Although we have not identified 


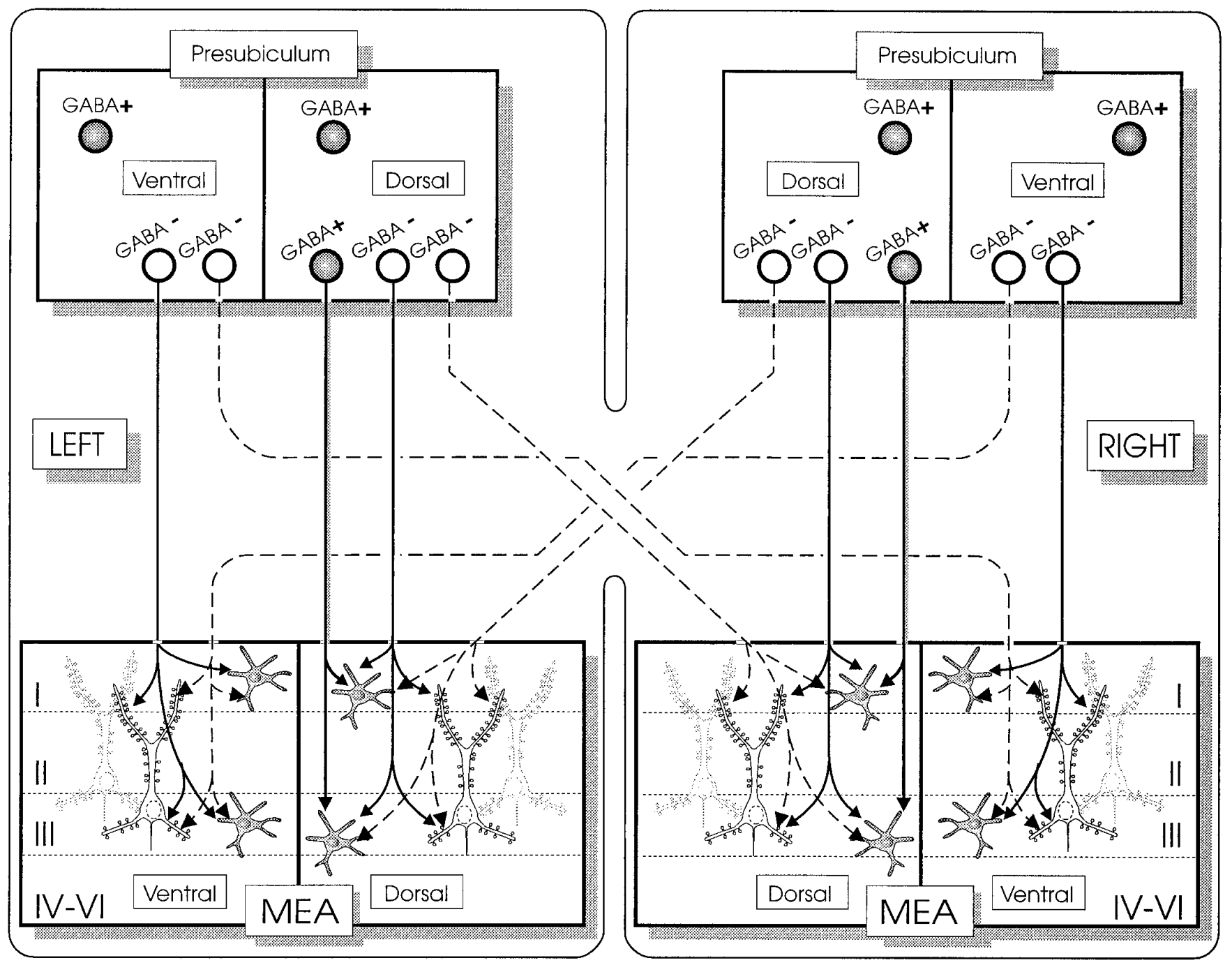

Figure 9. Proposed scheme of connectivity between the Presubiculum and the $M E A$, as derived from the results of combining antero- and retrograde tracing techniques, GABA immunocytochemistry, and electron microscopy. Our results demonstrate that $\sim 30-40 \%$ of all GABA-immunopositive neurons $(G A B A+$; filled circles with projection) in the dorsal presubiculum project to the ipsilateral dorsal MEA, where they terminate on dendritic shafts of interneurons (opaque cells) in layers I and III. No GABAergic neurons projecting to contralateral MEA are present. GABAergic projection neurons are absent in ventral presubiculum. Most GABAergic neurons in dorsal and ventral presubiculum $(G A B A+;$ filled circles without projection) are neurons without identified projections. Non-GABAergic neurons (GABA-; open circles with projection) in both the dorsal and ventral presubiculum project to ipsilateral or contralateral MEA, however, not to both areas. These projections follow a dorsal-to-ventral topography with regard to their termination in MEA; i.e., neurons in dorsal presubiculum project to dorsal MEA, whereas neurons in ventral presubiculum project to ventral MEA. These projections terminate in an almost equal ratio on both spines of principal neurons (transparent neurons) and dendritic shafts of interneurons (opaque neurons) in layers I and III. Roman numerals indicate the layers of MEA; LEFT and RIGHT indicate the two hemispheres of the brain.

any of the postsynaptic neurons, previous morphological and immunocytochemical studies have shown that entorhinal principal neurons are commonly spine-bearing, whereas entorhinal interneurons are almost spineless (Germroth et al., 1989; Lingenhöhl and Finch, 1991; Caballero-Bleda and Witter, 1994; Van Haeften et al., 1995a; Wouterlood et al., 1995a). This was corroborated by serial sectioning of large parts of dendrites that received synaptic contacts on their shafts. It was shown that these dendrites had most of the characteristics of interneurons; i.e., they were almost spine-free and received converging asymmetrical synapses (Fig. 8). Moreover, ongoing electron microscopical studies in our laboratory have shown that presubicular fibers target parvalbuminergic neurons in MEA (Wouterlood et al., 1996). Because this calcium-binding protein has been shown to coexist with GABA in cortical interneurons (Celio, 1986), presubicular fibers thus ap- pear to target GABAergic interneurons in MEA. As summarized in Figure 9, the majority of the presubicular fibers target principal neurons as well as interneurons in approximately equal percentages, a conclusion that confirms previous findings (CaballeroBleda and Witter, 1994). In contrast, the GABAergic projections from the presubiculum appear to selectively target entorhinal interneurons.

\section{Functional implications}

We propose that the presubiculum, through its massive projections to layers I and III of MEA, influences entorhinal layer III projection neurons, which give rise to projections to the hippocampal field CA1 and the subiculum (Amaral and Witter, 1995). Presubicular fibers may influence the firing of principal neurons either directly or by way of feedforward inhibition (cf. 
Finch et al., 1988). Moreover, the present study provides the first morphological evidence that GABAergic presubicular projection neurons may also exert disinhibitory effects on the entorhinal network, mediated by local GABAergic interneurons. Electrophysiological studies are needed to substantiate these anatomically based inferences and to elucidate their possible functional relevances. Concerning the latter issue, it should be stressed that the proposed disinhibitory circuits within the presubiculo-entorhinal connections are restricted, both with respect to their presubicular origin as well as their termination in MEA (cf. Caballero-Bleda and Witter, 1993). Based on cytoarchitectonic (Vogt and Miller, 1983), connectional (Swanson and Cowan, 1977; Vogt and Miller, 1983; Van Groen and Wyss, 1990b), and functional characteristics (Taube et al., 1990; Muller et al., 1996), it has been proposed that the presubiculum actually consists of two functionally different entities, the dorsal presubiculum or postsubiculum, and the ventral presubiculum or presubiculum proper. Differences between the dorsal and ventral parts of MEA have recently been reported regarding the vulnerability of layer III neurons to excessive stimulation. In rats, layer III neurons in the ventral part of MEA are more sensitive to application of various excitotoxins or otherwise induced limbic seizures (Du et al., 1995; Eid et al., 1995). Likewise, similarly positioned layer III neurons are among the first to show signs of degeneration in human temporal lobe epilepsy (Du et al., 1993). Although these different susceptibilities may reflect differences in the intrinsic networks or physiological characteristics of the constituting neuronal elements of dorsal versus ventral parts of MEA, it is tempting to suggest that a relation may exist with the presence or absence of presubicular GABAergic fibers.

\section{REFERENCES}

Allan CN, Crawford IL (1984) GABAergic agents in the medial septal nucleus affect hippocampal theta rhythm and acetylcholine utilization. Brain Res 322:261-267.

Amaral DG, Witter MP (1995) The hippocampal formation. In: The rat nervous system, 2nd Ed (Paxinos G, ed), pp 443-493. San Diego: Academic.

Beckstead RM (1978) Afferent connections of the entorhinal area in the rat as demonstrated by retrograde cell-labeling with horseradish peroxidase. Brain Res 152:249-264.

Buhl EH, Jones RSG (1993) Basket-like interneurons in layer II of the entorhinal cortex exhibit a powerful NMDA-mediated synaptic inhibition. Neurosci Lett 149:35-39.

Buijs RM, Van Vulpen EHS, Geffard M (1987) Ultrastructural localization of GABA in the supraoptic nucleus and neural lobe. Neuroscience 20:347-355.

Caballero-Bleda M, Witter MP (1993) Regional and laminar organization of projections from the presubiculum and parasubiculum to the entorhinal cortex. An anterograde tracing study in the rat. J Comp Neurol 328:115-129.

Caballero-Bleda M, Witter MP (1994) Projections from the presubiculum and parasubiculum to morphologically characterized entorhinalhippocampal projection neurons in the rat. Exp Brain Res 101:93-108.

Celio MR (1986) Parvalbumin in most gamma-amino-butyric acidcontaining neurons in the rat cerebral cortex. Science 231:995-997.

Colonnier M (1968) Synaptic patterns on different cell types in the different laminae of the cat visual cortex. An electron microscope study. Brain Res 9:268-287.

Dolleman-van-der-Weel MJ, Witter MP (1996) Projections from the nucleus reuniens thalami to the entorhinal cortex, hippocampal field CA1, and the subiculum in the rat arise from different populations of neurons. J Comp Neurol 364:637-650.

Du F, Whetsell WO, Abou-Khalil B, Blumenkopf B, Lothman EW,
Schwarcz R (1993) Preferential neuronal loss in layer III of the entorhinal cortex in patients with temporal lobe epilepsy. Epilepsy Res 16:223-233.

Du F, Eid T, Lothman EW, Köhler C, Schwarcz R (1995) Preferential neuronal loss in layer III of the medial entorhinal cortex in rat models of temporal lobe epilepsy. J Neurosci 10:6301-6313.

Eid T, Du F, Schwarcz R (1995) Differential neuronal vulnerability to amino-oxyacetate and quinolinate in the rat parahippocampal region. Neuroscience 68:645-656.

Eid T, Jorritsma-Byham B, Schwarcz R, Witter MP (1996) Afferents to the seizure-sensitive neurons in layer III of the medial entorhinal area: a tracing study in the rat. Exp Brain Res 109:209-218.

Finch DM, Tan AM, Isokawa-Akesson M (1988) Feedforward inhibition of the rat entorhinal cortex and subicular complex. J Neurosci 8:2213-2226.

Freund TF (1989) GABAergic septohippocampal neurons contain parvalbumin. Brain Res 478:375-381.

Germroth P, Schwerdtfeger WK, Buhl EH (1989) Morphology of identified entorhinal neurons projecting to the hippocampus. A light microscopical study combining retrograde tracing and intracellular injection. Neuroscience 30:683-691.

Gray EG (1959) Axo-somatic and axo-dendritic synapses of the cerebral cortex: an electron microscope study. J Anat 93:420-433.

Jones RSG (1993) Entorhinal-hippocampal connections: a speculative view of their function. Trends Neurosci 16:58-64.

Jones RSG, Buhl EH (1993) Basket-like interneurones in layer II of the entorhinal cortex exhibit a powerful NMDA-mediated synaptic excitation. Neurosci Lett 149:35-39.

Köhler C (1984) Morphological details of the projection from the presubiculum to the entorhinal area as shown with the novel PHA-L immunohistochemical tracing method in the rat. Neurosci Lett 45:285-290.

Köhler C (1985) Intrinsic projections of the retrohippocampal region in the rat brain. I. The subicular complex. J Comp Neurol 236:504-522.

Köhler C, Shipley MT, Srebro B, Harkmark W (1978) Some retrohippocampal afferents to the entorhinal cortex. Cells of origin as studied by the HRP method in the rat and mouse. Neurosci Lett 10:115-120.

Köhler C, Wu JY, Chan-Palay V (1985) Neurons and terminals in the retrohippocampal region in the rat's brain identified by anti-gammaaminobutyric acid and anti-glutamic acid decarboxylase immunocytochemistry. Anat Embryol (Berl) 173:35-44.

Lingenhöhl K, Finch DM (1991) Morphological characterization of rat entorhinal neurons in vivo: soma-dendritic structure and axonal domains. Exp Brain Res 84:57-74.

Muller RU, Ranck Jr JB, Taube JS (1996) Head direction cells: properties and functional significance. Curr Opin Neurobiol 6:196-206.

Paxinos G, Watson C (1986) The rat brain in stereotaxic coordinates, 2nd Ed. Sydney: Academic.

Peters A, Palay SL, Webster DeFH (1991) The fine structure of the nervous system: neurons and their supporting cells, 3rd Ed. Oxford: Oxford UP.

Pickel VM, Chan J (1995) Co-localization of angiotensin II and gammaaminobutyric acid in axon terminals in the rat subfornical organ. Neurosci Lett 193:89-92.

Ribak CE (1992) Local circuitry of GABAergic basket cells in the dentate gyrus. Epilepsy Res [Suppl] 7:29-47.

Segal M (1977) Afferents to the entorhinal cortex of the rat as studied by the method of retrograde transport of horseradish peroxidase. Exp Neurol 57:750-765.

Seress L, Ribak CE (1983) GABAergic cells in the dentate gyrus appear to be local circuit and projection neurons. Exp Brain Res 50:173-182.

Shipley MT (1975) The topographical and laminar organization of the presubiculum's projection to the ipsi- and contralateral entorhinal cortex in the guinea pig. J Comp Neurol 160:127-146.

Soriano E, Frotscher M (1993) GABAergic innervation of the rat fascia dentata: a novel type of interneuron in the granule cell layer with extensive axonal arborization in the molecular layer. J Comp Neurol 334:385-396.

Steward O (1976) Topographic organization of the projections from the entorhinal area to the hippocampal formation of the rat. J Comp Neurol 167:285-314.

Steward O, Scoville SA (1976) Cells of origin of entorhinal cortical afferents to the hippocampus and fascia dentata of the rat. J Comp Neurol 169:347-370. 
Swanson LW, Cowan WM (1977) An autoradiographic study of the organization of the efferent connections of the hippocampal formation in the rat. J Comp Neurol 172:49-84.

Szabat E, Soinila S, Häppölä O, Linnala A, Virtanen I (1992) A new monoclonal antibody against the GABA-protein conjugate shows immunoreactivity in sensory neurons in the rat. Neuroscience 47:409-420.

Taube JS, Muller RU, Ranck JB (1990) Head-direction cells recorded from the postsubiculum in freely moving rats. I. Description and quantitative analysis. J Neurosci 10:420-435.

Van der Linden S, Witter MP, Lopes da Silva FH (1996) Comparison of electrophysiology and morphology of layer III and layer II cells in the entorhinal cortex. Soc Neurosci Abstr 22:902.

Van Groen T, Wyss JM (1990a) The connections of presubiculum and parasubiculum in the rat. Brain Res 518:227-243.

Van Groen T, Wyss JM (1990b) The postsubicular cortex in the rat: characterization of the fourth region of the subicular cortex and its connections. Brain Res 529:165-177.

Van Haeften T, Jorritsma-Byham B, Witter MP (1995a) Quantitative morphological analysis of subicular terminals in the rat entorhinal cortex. Hippocampus 5:452-459.

Van Haeften T, Wouterlood FG, Jorritsma-Byham B, Witter MP (1995b) Light- and electron-microscopic demonstration of a presubicular GABAergic projection to the medial entorhinal cortex of the rat. Eur J Neurosci [Suppl] 8:153.

Vogt BA, Miller MW (1983) Cortical connections between rat cingulate cortex and visual, motor, and postsubicular cortices. J Comp Neurol 216:192-210.

Witter MP, Groenewegen HJ, Lopes da Silva FH, Lohman AHM (1989) Functional organization of the extrinsic and intrinsic circuitry of the parahippocampal region. Prog Neurobiol 33:161-253.

Wouterlood FG, Saldana E, Witter MP (1990) Projection from the nucleus reuniens thalami to the hippocampal region: light and electron microscopic tracing study in the rat with the anterograde tracer Phaseolus vulgaris-leucoagglutinin. J Comp Neurol 296:179-203.

Wouterlood FG, Pattiselanno A, Jorritsma-Byham B, Arts MPM, Meredith GE (1993) Connectional, immunocytochemical and ultrastructural characterization of neurons injected intracellularly in fixed brain tissue. In: IBRO handbook series, Vol 16, Methods in the neurosciences (Meredith GE, Arbuthnott GW, eds), pp 47-74. Chichester: Wiley.

Wouterlood FG, Härtig W, Brückner G, Witter MP (1995a) Parvalbumin-immunoreactive neurons in the entorhinal cortex of the rat: localization, morphology, connectivity and ultrastructure. J Neurocytol 24:135-153.

Wouterlood FG, Van Haeften T, Witter MP (1995b) Demonstration of a presubicular GABAergic projection to the medial entorhinal cortex of the rat. Soc Neurosci Abstr 21:428.

Wouterlood FG, Martin-Clemente B, Van Haeften T, Witter MP (1996) Presubicular axon terminals synapse with parvalbumin-immunoreactive interneurons in the medial entorhinal cortex of the rat. Soc Neurosci Abstr 22:903. 